\title{
Upper Bounds on the Bit-Error Rate of Optimum Combining in Wireless Systems
}

\author{
Jack H. Winters, Fellow, IEEE, and Jack Salz, Member, IEEE
}

\begin{abstract}
This paper presents upper bounds on the bit-error rate (BER) of optimum combining in wireless systems with multiple cochannel interferers in a Rayleigh fading environment. We present closed-form expressions for the upper bound on the bit-error rate with optimum combining, for any number of antennas and interferers, with coherent detection of BPSK and QAM signals, and differential detection of DPSK. We also present bounds on the performance gain of optimum combining over maximal ratio combining. These bounds are asymptotically tight with decreasing BER, and results show that the asymptotic gain is within $2 \mathrm{~dB}$ of the gain as determined by computer simulation for a variety of cases at a $10^{-3}$ BER. The closed-form expressions for the bound permit rapid calculation of the improvement with optimum combining for any number of interferers and antennas, as compared with the CPU hours previously required by Monte Carlo simulation. Thus these bounds allow calculation of the performance of optimum combining under a variety of conditions where it was not possible previously, including analysis of the outage probability with shadow fading and the combined effect of adaptive arrays and dynamic channel assignment in mobile radio systems.
\end{abstract}

Index Terms - Bit-error rate, optimum combining, Rayleigh fading, smart antennas.

\section{INTRODUCTION}

A NTENNA arrays with optimum combining combat multipath fading of the desired signal and suppress interfering signals, thereby increasing both the performance and capacity of wireless systems. With optimum combining, the received signals are weighted and combined to maximize the signal-tointerference-plus-noise ratio (SINR) at the receiver. Optimum combining yields superior performance over maximal ratio combining, whereby the signals are combined to maximize signal-to-noise ratio, in interference-limited systems. However, while with maximal ratio combining the bit-error rate can be expressed in closed form [1], with optimum combining a closed-form expression is available only with one interferer [2], [3]. With multiple interferers, Monte Carlo simulation has been used [3]-[5], but this requires on the order of CPU hours even with just a few interferers. Thus the improvement of optimum combining has only been studied for a few simple

Paper approved by N. C. Beaulieu, the Editor for Wireless Communication Theory of the IEEE Communications Society. Manuscript received September 21, 1993; revised November 28, 1996. This paper was presented in part at the 1994 IEEE Vehicular Technology Conference, Stockholm, Sweden, June 8-10, 1994.

J. H. Winters is with AT\&T Labs-Research, Red Bank, NJ 07701 USA.

J. Salz, retired, was with AT\&T Labs-Research, Crawford Hill Laboratory, Holmdel, NJ 07733 USA.

Publisher Item Identifier S 0090-6778(98)09388-X.

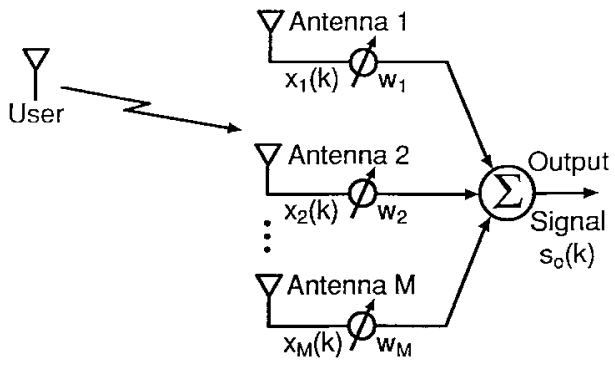

Fig. 1. Block diagram of an $M$-element adaptive array.

cases, and detailed comparisons (e.g., in terms of outage probability) have not been done.

In [6], we showed that, with $M$ antenna elements, the received signals can be combined to eliminate $L(L<M)$ interferers in the output signal while obtaining an $M-L$ diversity improvement, i.e., the performance of maximal ratio combining with $M-L$ antennas and no interference. However, this "zero-forcing" solution gives far lower output SINR than optimum combining in most cases of interest and cannot be used when $L \geq M$.

In this paper we present a closed-form expression for the upper bound on the bit-error rate (BER) with optimum combining in wireless systems. We assume flat fading across the channel and independent Rayleigh fading of the desired and interfering signals at each antenna. ${ }^{1}$ Equations are presented for the upper bound on the BER for coherent detection of quadrature amplitude modulated (QAM) and binary phase-shift-keyed (BPSK) signals, and for differential detection of differential phase-shift-keyed (DPSK) signals. From these equations, a lower bound on the improvement of optimum combining over maximal ratio combining is derived.

In Section II we derive the upper bound on the BER. In Section III we compare the upper bound to Monte Carlo simulation results. A summary and conclusions are presented in Section IV.

\section{UPPER BOUND DERIVATION}

Fig. 1 shows a block diagram of an $M$-element adaptive array. The complex baseband signal received by the $i$ th antenna element in the $k$ th symbol interval $x_{i}(k)$ is multiplied by a controllable complex weight $w_{i}$ and the weighted signals are summed to form the array output signal $s_{\circ}(k)$.

\footnotetext{
${ }^{1}$ As shown in [7], the gain of optimum combining is not significantly degraded with fading correlation up to about 0.5 . Thus our bounds, based on independent fading, are reasonably accurate and useful even in environments with fading correlation up to this level.
} 
With optimum combining, the weights are chosen to maximize the output SINR, which also minimizes the mean-square error (MSE), which is given by [8]

$$
\text { MSE }=\left(1+\boldsymbol{u}_{d}^{\dagger} \boldsymbol{R}_{n n}^{-1} \boldsymbol{u}_{d}\right)^{-1}
$$

where $\boldsymbol{R}_{n n}$ is the received interference-plus-noise correlation matrix given by

$$
\boldsymbol{R}_{n n}=\sigma^{2} \boldsymbol{I}+\sum_{j=1}^{L} \boldsymbol{u}_{j} \boldsymbol{u}_{j}^{\dagger}
$$

$\sigma^{2}$ is the noise power, $I$ is the identity matrix, $\boldsymbol{u}_{d}$ and $\boldsymbol{u}_{j}$ are the desired and $j$ th interfering signal propagation vectors, respectively, and the superscript $\dagger$ denotes complex conjugate transpose. Here we have assumed the same average received power for the desired signal at each antenna (that is, microdiversity rather than macrodiversity) and that the noise and interfering signals are uncorrelated, and without loss of generality, have normalized the received signal power, averaged over the fading, to 1 . Note that the MSE varies at the fading rate.

For coherent detection of BPSK or QAM, the BER is bounded by [9]

$$
P_{e} \leq e^{\left(1 / \sigma_{c}^{2}\right)} E\left[e^{(-1 / \mathrm{MSE})}\right]=e^{\left(\left(1 / \sigma_{c}^{2}\right)-1\right)} E\left[e^{-\boldsymbol{u}_{d}^{\dagger} \boldsymbol{R}_{n n}^{-1} \boldsymbol{u}_{d}}\right]
$$

where now the expected value is taken over the fading parameters of the desired and interfering signals, and $\sigma_{c}^{2}$ is the variance of the BPSK or QAM symbol levels (e.g., $\sigma_{c}^{2}=1$ and 2 for BPSK and quaternary phase-shift keying (QPSK), respectively). For differential detection of DPSK, assuming Gaussian noise and interference, ${ }^{2}$ the BER is given by [1]

$$
P_{e}=\frac{1}{2} E\left[e^{-\boldsymbol{u}_{d}^{\dagger} \boldsymbol{R}_{n n}^{-1} \boldsymbol{u}_{d}}\right] .
$$

Thus the BER expression for both cases differs only by a constant, and we will now consider the term $E\left[e^{-\boldsymbol{u}_{d}^{\dagger} \boldsymbol{R}_{n n}^{-1} \boldsymbol{u}_{d}}\right]$. As shown in the Appendix, this term can be upper-bounded by

$$
E\left[e^{-\boldsymbol{u}_{d}^{\dagger} \boldsymbol{R}_{n n}^{-1} \boldsymbol{u}_{d}}\right] \leq E\left[\prod_{n=1}^{M} \lambda_{n}\right]=E\left[\left|\boldsymbol{R}_{n n}\right|\right]
$$

where $\left|\boldsymbol{R}_{n n}\right|$ denotes the determinant of $\boldsymbol{R}_{n n}$, and $\lambda_{n}$ is the $n$th eigenvalue of $\boldsymbol{R}_{n n}$.

Since (5) is the key inequality in our bound (and is the only inequality we use in determining the bound for differential detection of DPSK), let us examine its accuracy. The bound is tight if $\lambda_{n} \ll 1$, and since the $\lambda_{n}$ 's are proportional to the interference signal powers, the bound is tight for large received SINR, i.e., low BER's. Although for all cases $\left(1+\left(1 / \lambda_{n}\right)\right)^{-1}<1$ and thus BER $<0.5$, for $\lambda_{n}>1$ the BER as given by the bound may exceed 0.5 . Thus with small received SINR, occasionally BER's greater than 0.5 may be averaged into the average BER, reducing the tightness of

\footnotetext{
${ }^{2}$ Since the stronger the interference, the more that optimum combining suppresses it, with the Gaussian assumption we overestimate the probability of strong interference. Note that this is consistent with the derivation of an upper bound on the BER.
}

the bound. Also, note that with only noise at the receiver, $\lambda_{n}=\sigma_{n}^{2}$, where $\sigma_{n}^{2}$ is the variance of the noise normalized to the received desired signal power, and from (4) and (5)

$$
P_{e} \leq \frac{\left(\sigma_{n}^{2}\right)^{M}}{2}=\frac{1}{2 \rho^{M}}
$$

where $\rho$ is the received SINR, while the actual BER is $1 / 2(1+\rho)^{M}[1]$. Thus even without interference, the bound differs from the actual BER, and this difference increases as the received SINR decreases.

Let us consider the case of interference only. In this case, $\left|\boldsymbol{R}_{n n}\right|$, which is given by (2), may also be expressed as

$$
\left|\boldsymbol{R}_{n n}\right|=\left|\boldsymbol{Q}^{\dagger} \boldsymbol{Q}\right|=\sum \pm \boldsymbol{D}_{1}^{\dagger} \boldsymbol{D}_{m 1} \boldsymbol{D}_{2}^{\dagger} \boldsymbol{D}_{m 2} \cdots \boldsymbol{D}_{M}^{\dagger} \boldsymbol{D}_{m_{M}}
$$

where $\boldsymbol{Q}=\left(\boldsymbol{D}_{1}, \cdots, \boldsymbol{D}_{M}\right), \boldsymbol{D}_{m}=\left(\left(\boldsymbol{u}_{1}\right)_{m} \cdots\left(\boldsymbol{u}_{L}\right)_{m}\right)^{T}$, $\left(\boldsymbol{u}_{j}\right)_{m}$ is the $m$ th element of $\boldsymbol{u}_{j}$, the sum is extended over all $M$ ! permutations of the $\boldsymbol{D}_{m}$ 's, $\boldsymbol{D}_{m_{i}}$ is the $i$ th element of the permutation of the $D_{m}$ 's, the "+" sign is assigned for even permutations (i.e., an even number of swapping of $\boldsymbol{D}_{m}$ 's in the permutation), and the "-" sign for odd permutations. Now

$$
E\left[\boldsymbol{D}_{m}^{\dagger} \boldsymbol{D}_{m}\right]=\sum_{j=1}^{L} \sigma_{j}^{2}
$$

where $\sigma_{j}^{2}$ is the average power of the $j$ th interferer normalized to the desired signal power, and

$$
E\left[\boldsymbol{D}_{m}^{\dagger} \boldsymbol{D}_{n} \boldsymbol{D}_{n}^{\dagger} \boldsymbol{D}_{m}\right]=\sum_{j=1}^{L} \sigma_{j}^{4}
$$

Similarly, from (7), it can be shown that

$$
\begin{aligned}
E\left[\left|\boldsymbol{Q}^{\dagger} \boldsymbol{Q}\right|\right]= & \sum_{q} \alpha_{q}^{(M)}\left(\sum_{j=1}^{L} \sigma_{j}^{2}\right)^{M-\sum_{k} i_{k} l_{l_{k}}}\left(\sum_{j=1}^{L} \sigma_{j}^{2 i_{1}}\right)^{l_{1}} \\
& \cdot\left(\sum_{j=1}^{L} \sigma_{j}^{2 i_{2}}\right)^{l_{2}} \cdots
\end{aligned}
$$

where the sum is over all sets of positive integers $i_{k}$ and $l_{k}$ that exist such that $M \geq \cdots>i_{2}>i_{1}$, with $\sum_{k} i_{k} l_{k} \leq M$. For example, when $M=5$, there are 6 sets of $\left\{i_{k}, l_{k}\right\}$ such that $\sum_{k} i_{k} l_{k} \leq M$ (see Table I). All sets are of the form $\left\{i_{1}, l_{1}\right\}$, e.g., $\left\{i_{1}=3, l_{1}=1\right\}$ for $3 \cdot 1<5$, except for the set $\left\{i_{1}=2, l_{1}=1, i_{2}=3, l_{2}=1\right\}$ for $2 \cdot 1+3 \cdot 1=5$. $\alpha_{q}^{(M)}$ is an integer coefficient corresponding to the $q$ th set with $M$ antennas. Note that $\alpha_{q}^{(M)}$ is obtained by summing the coefficients ( \pm 1 's) for similar terms in $E\left[\left|\boldsymbol{Q}^{\dagger} \boldsymbol{Q}\right|\right] . \alpha_{q}^{(M)}$ can be determined as shown below.

Since $\sum_{j=1}^{L} \sigma_{j}^{2}=1 / \rho$, and $\alpha_{q}^{(M)}=1$ when $\sum_{k} i_{k} l_{k}=0$, (10) can also be expressed as

$$
\begin{aligned}
E\left[\left|\boldsymbol{Q}^{\dagger} \boldsymbol{Q}\right|\right]=\rho^{-M} & {\left[1+\sum_{q} \alpha_{q}^{(M)}\left(\sum_{j=1}^{L}\left(\rho \sigma_{j}^{2}\right)^{i_{1}}\right)^{\boldsymbol{l}_{1}}\right.} \\
& \left.\cdot\left(\sum_{j=1}^{L}\left(\rho \sigma_{j}^{2}\right)^{i_{2}}\right)^{l_{2}} \ldots\right]
\end{aligned}
$$


TABLE I

\begin{tabular}{c|c|c|c|c|c}
\multicolumn{7}{c}{ TABLE I } \\
\multicolumn{1}{c}{ VALUES OF $\alpha_{q}^{(M)}$ FOR $M=2$ TO 5} \\
\hline \hline$M$ & $i_{1}$ & $l_{1}$ & $i_{2}$ & $l_{2}$ & $\alpha_{q}^{(M)}$ \\
& & & & & \\
\hline 2 & 2 & 1 & & & -1 \\
& & & & & \\
\hline 3 & 2 & 1 & & & -3 \\
& 3 & 1 & & & +2 \\
& & & & & \\
\hline 4 & 2 & 1 & & & -6 \\
& 2 & 2 & & & +3 \\
& 3 & 1 & & & +8 \\
& 4 & 1 & & & -6 \\
& & & & & \\
\hline 5 & 2 & 1 & & & -10 \\
& 2 & 2 & & & +15 \\
& 3 & 1 & & & +20 \\
& 2 & 1 & 3 & 1 & -20 \\
& 4 & 1 & & & -30 \\
& 5 & 1 & & & +24 \\
\hline \hline & & & & & \\
\hline
\end{tabular}

where now $M \geq \cdots>i_{2}>i_{1}>1$.

To determine the $\alpha_{q}^{(M)}$, s, first note that if $\sigma_{j}^{2}=\sigma^{2}, j=$ $1, \cdots, L$, then $\sum_{j=1}^{L} \sigma_{j}^{2 k}=L \sigma^{2 k}$, and (11) becomes

$$
E\left[\left|\boldsymbol{Q}^{\dagger} \boldsymbol{Q}\right|\right]=\left(L^{M}+\sum_{k=2}^{M} \beta_{k} L^{M-k+1}\right) \sigma^{2 M}
$$

where the $\beta_{k}$ 's and the $\alpha_{q}^{(M)}$, s can be seen to be closely related. From [6], $P_{e}=0$ for $L<M$, and thus the $\beta_{k}$ 's are the coefficients of the $M$ th-order polynomial in $L$, $L(L-1)(L-2) \cdots(L-M+1)$. This result is not only useful when all interferers have equal power, but also serves as a consistency check on our calculated values of $\alpha_{q}^{(M)}$.

The values of $\alpha_{q}^{(M)}$ were generated using a computer program to examine every permutation in (7) for given $M$. The number of each type of $i_{1}, l_{1}, i_{2}, l_{2}, \cdots$ term was calculated to determine $\alpha_{q}^{(M)}$. Tables I and II list these values for $M=2-7$. Note that only $i_{1}$ and $l_{1}$ terms exist for $M \leq 4$, and $i_{2}$ and $l_{2}$ terms also exist for $5 \leq M$. Values for $\alpha_{q}^{(M)}$ for higher $M$ can also be easily calculated. However, since the amount of computer time to generate the values of $\alpha_{q}^{(M)}$ increases exponentially with $M$, our program could only generate these values in a reasonable amount of computer time for up to $M=10$ (where a hundred CPU hours on a SPARCstation20 would be required).

From (3), the upper bound on the BER with coherent detection of BPSK or QAM is now given by

$$
\begin{aligned}
P_{e} \leq e^{\left.\left(1 / \sigma_{c}^{2}\right)-1\right)} \rho^{-M} & {\left[1+\sum_{q} \alpha_{q}^{(M)}\left(\sum_{j=1}^{L}\left(\rho \sigma_{j}^{2}\right)^{i_{1}}\right)^{l_{1}}\right.} \\
& \left.\cdot\left(\sum_{j=1}^{L}\left(\rho \sigma_{j}^{2}\right)^{i_{2}}\right)^{l_{2}} \cdots\right]
\end{aligned}
$$

\begin{tabular}{c|c|c|c|c|c}
\multicolumn{6}{c}{ TABLE II } \\
\multicolumn{1}{c}{ VALUES OF $\alpha_{q}^{(M)}$ FOR $M=6$ AND 7} \\
\hline \hline$M$ & $i_{1}$ & $l_{1}$ & $i_{2}$ & $l_{2}$ & $\alpha_{q}^{(M)}$ \\
& & & & & \\
\hline 6 & 2 & 1 & & & -15 \\
& 2 & 2 & & & +45 \\
& 2 & 3 & & & -15 \\
& 3 & 1 & & & +40 \\
& 3 & 2 & & & +40 \\
& 4 & 1 & & & -90 \\
& 5 & 1 & & & +144 \\
& 6 & 1 & & & -120 \\
& 2 & 1 & 3 & 1 & -120 \\
& 2 & 1 & 4 & 1 & +90 \\
& & & & & \\
\hline 7 & 2 & 1 & & & -21 \\
& 2 & 2 & & & +105 \\
& 2 & 3 & & & -105 \\
& 3 & 1 & & & +70 \\
& 3 & 2 & & & +280 \\
& 4 & 1 & & & -210 \\
& 5 & 1 & & & +504 \\
6 & 1 & & & -840 \\
7 & 1 & & & +720 \\
2 & 1 & 3 & 1 & -420 \\
2 & 1 & 4 & 1 & +630 \\
2 & 1 & 5 & 1 & -504 \\
3 & 1 & 4 & 1 & -420 \\
2 & 2 & 3 & 1 & +210 \\
& & & & & \\
\hline \hline & & & &
\end{tabular}

and from (4), the upper bound on the BER with differential detection of DPSK is given by

$$
\begin{aligned}
P_{e} \leq \frac{1}{2} \rho^{-M}\left[1+\sum_{q} \alpha_{q}^{(M)}\right. & \left(\sum_{j=1}^{L}\left(\rho \sigma_{j}^{2}\right)^{i_{1}}\right)^{l_{1}} \\
& \left.\cdot\left(\sum_{j=1}^{L}\left(\rho \sigma_{j}^{2}\right)^{i_{2}}\right)^{l_{2}} \cdots\right] .
\end{aligned}
$$

For the case of noise with $L$ interferers, consider the noise as an infinite number of weak interferers with total power equal to the noise. That is, let

$$
\sigma_{j}^{2}=\frac{\sigma_{n}^{2}}{K-L}, \quad j=L+1, \cdots, K,
$$

and let $K \rightarrow \infty$. Then, $\rho=\left(\sum_{j=1}^{L} \sigma_{j}^{2}+\sigma_{n}^{2}\right)^{-1}$, and

$$
\lim _{K \rightarrow \infty}\left[\left(\sum_{j=1}^{K} \sigma_{j}^{2 i_{k}}\right)^{l_{k}}\right]=\left[\left(\sum_{j=1}^{L} \sigma_{j}^{2 i_{k}}\right)^{l_{k}}\right]
$$

for $i_{k}>1$. Therefore, with noise, the BER bound is the same as in (13) and (14), but with $\rho$ including the noise. In this case, if we define the received desired signal-to-noise ratio as $\Gamma_{d}=\sigma_{n}^{-2}$ and the $j$ th interferer signal-to-noise ratio as 
$\Gamma_{j}=\sigma_{j}^{2} / \sigma_{n}^{2}$, then (14) becomes [similarly for (13)]

$$
\begin{gathered}
P_{e} \leq \frac{1}{2 \rho^{M}}\left[1+\sum_{q} \alpha_{q}^{(M)}\left(\sum_{j=1}^{L}\left(\frac{\Gamma_{j}}{\sum_{j=1}^{L} \Gamma_{j}+1}\right)^{i_{1}}\right)^{l_{1}}\right. \\
\left.\cdot\left(\sum_{j=1}^{L}\left(\frac{\Gamma_{j}}{\sum_{j=1}^{L} \Gamma_{j}+1}\right)^{i_{2}}\right)^{l_{2}} \cdots\right] .
\end{gathered}
$$

Since $\frac{1}{2 \rho^{M}}$ is the bound with maximal ratio combining, the term in the brackets is the improvement of optimum combining over maximal ratio combining based on the BER bound. Defining the gain of optimum combining as the reduction in the required $\rho$ for a given BER, from (17), this gain in decibels is given by

$$
\begin{aligned}
& \text { Gain (dB) } \\
& \begin{aligned}
=-\frac{10}{M} \log _{10} & {\left[1+\sum_{q} \alpha_{q}^{(M)}\left(\sum_{j=1}^{L}\left(\frac{\Gamma_{j}}{\sum_{j=1}^{L} \Gamma_{j}+1}\right)^{i_{1}}\right)^{l_{1}}\right.} \\
& \left.\cdot\left(\sum_{j=1}^{L}\left(\frac{\Gamma_{j}}{\sum_{j=1}^{L} \Gamma_{j}+1}\right)^{i_{2}}\right)^{l_{2}} \cdots\right] \cdot
\end{aligned}
\end{aligned}
$$

This gain is therefore independent of the desired signal power (because the bound is asymptotically tight as $\rho \rightarrow \infty$ ). However, this is the gain of the BER bound with optimum combining over the BER bound with maximal ratio combining. Since the required $\rho$ for a given BER with maximal ratio combining is less than the bound, the true gain may differ from (18) and to obtain a bound on the gain, the gain in (18) must be reduced accordingly. For example, with differential detection of DPSK, to obtain a bound the gain given in (18) is reduced by the factor $(\rho /(1+\rho))^{M}$. Note that as $\rho \rightarrow \infty$, this factor reduces to one and the gain approaches (18). Thus we will refer to (18) as the asymptotic gain.

\section{COMParison to EXACt Theory AND Simulation}

In this section, we compare the bound to theoretical results for $L=1$ and simulation results for $L \geq 2$.

Fig. 2 compares theoretical results (from [1]-[3]) for the gain to the asymptotic gain (18) versus BER with coherent detection of BPSK. Results are generated for $M=2$ and 5 , and $\Gamma_{1}=3$ and $10 \mathrm{~dB}$. In all cases the gain monotonically decreases to the asymptotic gain as the BER decreases. The gain approaches the asymptotic gain more slowly with decreasing BER for larger $M$ and also, at low BER's, the accuracy of the asymptotic gain decreases with higher $\Gamma_{1}$. Thus the accuracy of the asymptotic gain decreases as the $\rho$ required for a given BER with optimum combining decreases, as predicted by the approximation in Section II.

Fig. 3 compares theoretical and Monte Carlo simulation [5] results for the gain to the asymptotic gain with $M=2$ and $L=1,2$, and 6 . Results are plotted versus $\Gamma_{j}$, where all $L$ interferers have equal power, for coherent detection of BPSK

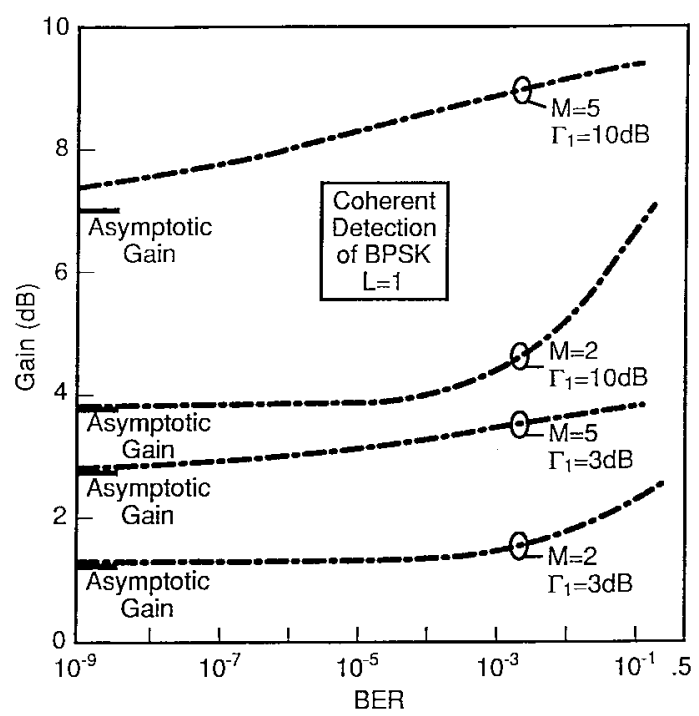

Fig. 2. Gain versus BER for coherent detection of BPSK-comparison of analytical results to the asymptotic gain.

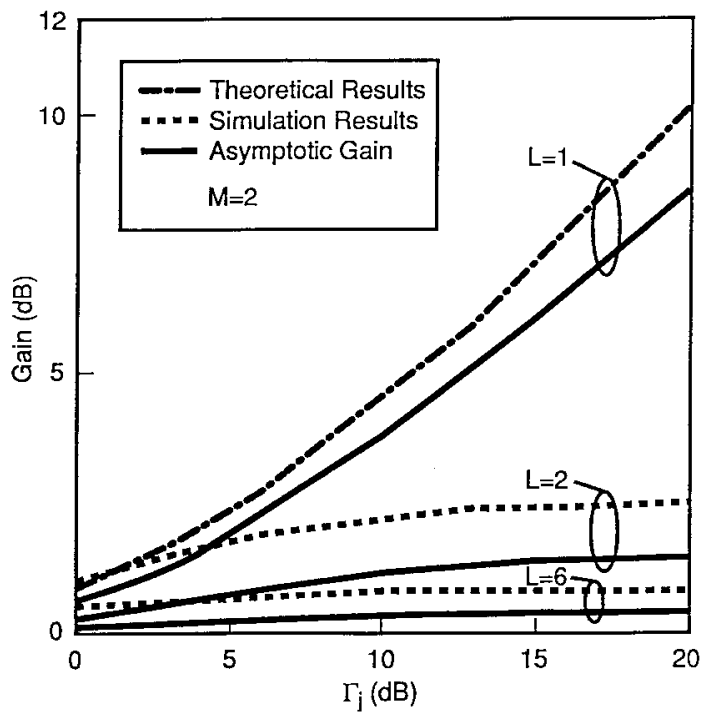

Fig. 3. Gain with $M=2$ for 1,2 , and 6 equal-power interferers versus signal-to-noise ratio of each interferer-comparison of analytical and Monte Carlo simulation results with coherent detection of BPSK [5] to the asymptotic gain.

at a $10^{-3}$ BER. $^{3}$ In all cases, the asymptotic gain has the same shape as the gain and is within $1.7 \mathrm{~dB}$ for $L=1$, $1.0 \mathrm{~dB}$ for $L=2$, and $0.4 \mathrm{~dB}$ for $L=6$. Since optimum combining gives the largest gain when the interference power is concentrated in one interferer and the least gain when the interference power is equally divided among many interferers, $L=1$ and $L=6$ represent the best and worst cases for the gain in an interference-limited cellular system. Thus from the results in Fig. 3, we would expect the asymptotic gain to be within $0.4-1.7 \mathrm{~dB}$ of the actual gain for all cases in cellular systems with $M=2$.

\footnotetext{
${ }^{3}$ This BER was used because the results in [5] were obtained for this BER. As shown in [5], the gain does not change significantly for BER's between $10^{-2}$ and $10^{-3}$, the range of interest in most mobile radio systems.
} 


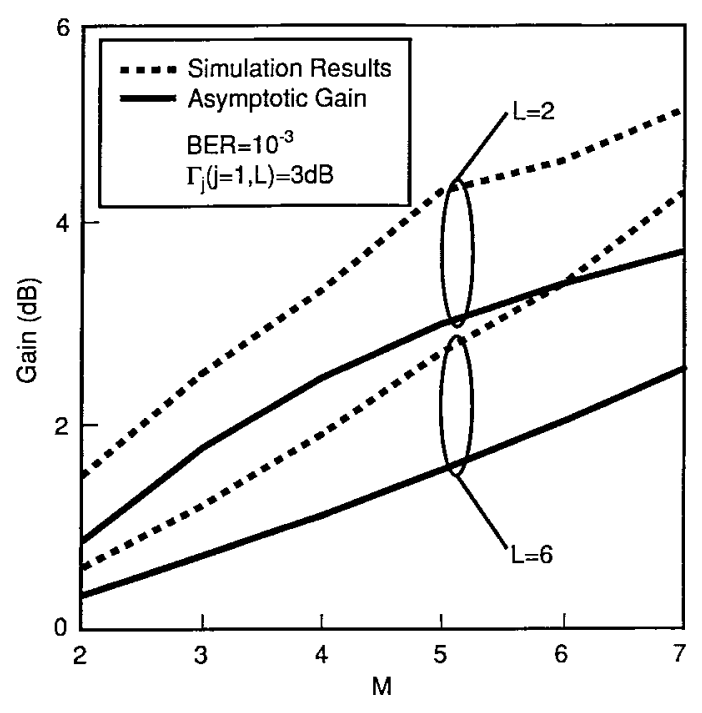

Fig. 4. Gain versus $M$ with two and six equal power interferers-comparison of Monte Carlo simulation results with coherent detection of BPSK [3] to the asymptotic gain.

Now, consider the lower bound on the gain obtained from the BER bound (17), as compared to the asymptotic gain. Without interference, differential detection of DPSK with maximal ratio combining and $M=2$ requires $\rho \approx 13.3 \mathrm{~dB}$ (theoretically [10]) for a $10^{-3}$ BER, while the BER bound (17) gives $\rho \approx 13.5 \mathrm{~dB}$. Thus the lower bound on the gain (from (17)) at a $10^{-3} \mathrm{BER}$ is $0.2 \mathrm{~dB}$ less than the asymptotic gain for any interference scenario-in particular, the lower bound on the gain is $0.2 \mathrm{~dB}$ less than the results shown in Fig. 3. Similarly, coherent detection of BPSK with maximal ratio combining and $M=2$ requires $\rho \approx 11.1 \mathrm{~dB}$ for a $10^{-3}$ BER, while the BER bound (13) gives $15.0 \mathrm{~dB}$. Thus the bound is most accurate with differential detection of DPSK and low BER's.

Fig. 4 compares Monte Carlo simulation results [3] for the gain to the asymptotic gain for $L=2$ and 6 . Results are plotted versus $M$ with $\Gamma_{j}=3 \mathrm{~dB}$ for all interferers and coherent detection of BPSK at a $10^{-3}$ BER. Again the asymptotic gain has the same shape as the simulation results. The cases include both many more interferers than antennas and many more antennas than interferers, but in all cases the asymptotic gain is within $1.8 \mathrm{~dB}$ of simulation results.

\section{CONCLUSIONS}

In this paper we have presented upper bounds on the biterror rate (BER) of optimum combining in wireless systems with multiple cochannel interferers in a Rayleigh fading environment. We presented closed-form expressions for the upper bound on the bit-error rate with optimum combining, for any number of antennas and interferers, with coherent detection of BPSK and QAM signals, and differential detection of DPSK. We also presented bounds on the performance gain of optimum combining over maximal ratio combining and showed that these bounds are asymptotically tight with decreasing BER. Results showed that the asymptotic gain is within $2 \mathrm{~dB}$ of the gain as determined by computer simulation for a variety of cases at a $10^{-3}$ BER. These cases include interference scenarios that cover the range of worst to best cases for the gain of optimum combining in cellular systems with $M=2$. The bound is most accurate with differential detection of DPSK and high SINR, corresponding to low BER and a few antennas. Because of the $2-\mathrm{dB}$ accuracy, the bound is most useful where the optimum combining improvement is the largest, which is the case of most interest. The closedform expression for the bound permits rapid calculation of the improvement with optimum combining for any number of interferers and antennas, as compared with the CPU hours previously required by Monte Carlo simulation. These bounds allow calculation of the performance of optimum combining under a variety of conditions where it was not possible previously, including analysis of the outage probability with shadow fading and the combined effect of adaptive arrays and dynamic channel assignment in mobile radio systems.

\section{APPENDIX}

Diagonalizing $\boldsymbol{R}_{n n}$ by a unitary transformation $\Psi$, we obtain

$$
\boldsymbol{R}_{n n}=\psi^{\dagger} \operatorname{diag}\left(\lambda_{1} \cdots \lambda_{M}\right) \psi
$$

where $\operatorname{diag}(\cdot)$ denotes an $M \times M$ matrix with nonzero elements only on the diagonal, or

$$
\boldsymbol{R}_{n n}^{-1}=\psi^{\dagger} \operatorname{diag}\left(\lambda_{1}^{-1} \cdots \lambda_{M}^{-1}\right) \psi
$$

and

$$
\boldsymbol{u}_{d}^{\dagger} \boldsymbol{R}_{n n}^{-1} \boldsymbol{u}_{d}=\boldsymbol{u}_{d}^{\dagger} \psi^{\dagger} \operatorname{diag}\left(\lambda_{1}^{-1} \cdots \lambda_{M}^{-1}\right) \psi \boldsymbol{u}_{d}
$$

Let

$$
\boldsymbol{C}=\left[c_{1} \cdots c_{M}\right]^{T}=\psi \boldsymbol{u}_{d}
$$

Then

and

$$
\boldsymbol{u}_{d}^{\dagger} \boldsymbol{R}_{n n}^{-1} \boldsymbol{u}_{d}=\sum_{n=1}^{M} \frac{\left|c_{n}\right|^{2}}{\lambda_{n}}
$$

$$
\begin{aligned}
E\left[e^{-\boldsymbol{u}_{d}^{\dagger} \boldsymbol{R}_{n n}^{-1} \boldsymbol{u}_{d}}\right] & =E\left[\exp \left(-\sum_{n=1}^{M} \frac{\left|c_{n}\right|^{2}}{\lambda_{n}}\right)\right] \\
& =E\left[\prod_{n=1}^{M} \exp \left(-\frac{\left|c_{n}\right|^{2}}{\lambda_{n}}\right)\right] .
\end{aligned}
$$

Since with independent, Rayleigh fading at each antenna, the elements of $\boldsymbol{u}_{d}$ are independent and identically distributed (i.i.d.) complex Gaussian random variables, the elements of $\boldsymbol{C}$ are also i.i.d. complex Gaussian random variables with the same mean and variance. Furthermore, the $\lambda_{n}$ 's are independent of the $c_{n}$ 's. Thus we can average over the desired and interfering signal vectors separately, i.e.,

$$
E\left[\prod_{n=1}^{M} \exp \left(-\frac{\left|c_{n}\right|^{2}}{\lambda_{n}}\right)\right]=E_{\lambda}\left[\prod_{n=1}^{M} E_{c_{n}}\left[\exp \left(-\frac{\left|c_{n}\right|^{2}}{\lambda_{n}}\right)\right]\right]
$$


Since the $c_{n}$ 's are complex Gaussian random variables with zero mean and unit variance

$$
E_{C_{n}}\left[\exp \left(-\frac{\left|c_{n}\right|^{2}}{\lambda_{n}}\right)\right]=\frac{1}{1+\frac{1}{\lambda_{n}}}
$$

and

$$
E\left[e^{-\boldsymbol{u}_{d}^{\dagger} \boldsymbol{R}_{n n}^{-1} \boldsymbol{u}_{d}}\right]=E_{\lambda}\left[\prod_{n=1}^{M}\left(\frac{1}{1+\frac{1}{\lambda_{n n}}}\right)\right] .
$$

Since the $\lambda_{n}$ 's are nonnegative

$$
\frac{1}{1+\frac{1}{\lambda_{n}}} \leq \lambda_{n}
$$

and, therefore,

$$
E\left[e^{-\boldsymbol{u}_{d}^{\dagger} \boldsymbol{R}_{n n}^{-1} \boldsymbol{u}_{d}}\right] \leq E_{\lambda}\left[\prod_{n=1}^{M} \lambda_{n}\right]=E_{\lambda}\left[\left|\boldsymbol{R}_{n n}\right|\right]
$$

where $\left|\boldsymbol{R}_{n n}\right|$ denotes the determinant of $\boldsymbol{R}_{n n}$.

\section{REFERENCES}

[1] W. C. Jakes Jr. et al., Microwave Mobile Communications. New York: Wiley, 1974.

[2] V. M. Bogachev and I. G. Kiselev, "Optimum combining of signals in space-diversity reception," Telecommun. Radio Eng., vol. 34/35, no. 10, pp. 83, Oct. 1980.

[3] J. H. Winters, "Optimum combining in digital mobile radio with cochannel interference," IEEE J. Select. Areas Commun., vol. SAC-2, no. 4, July 1984.

[4] _ "Optimum combining for indoor radio systems with multiple users,” IEEE Trans. Commun., vol. COM-35, no. 11, Nov. 1987.

[5] _ "Signal acquisition and tracking with adaptive arrays in the digital mobile radio system IS-54 with flat fading," IEEE Trans. Veh. Technol., Nov. 1993.

[6] J. H. Winters, J. Salz, and R. D. Gitlin, "The impact of antenna diversity on the capacity of wireless communication systems," IEEE Trans. Commun., Apr. 1994.
[7] J. Salz and J. H. Winters, "Effect of fading correlation on adaptive arrays in digital wireless communications," IEEE Trans. Veh. Technol., vol. 43, pp. 1049-1057, Nov. 1994.

[8] R. A. Monzingo and T. W. Miller, Introduction to Adaptive Arrays. New York: Wiley, 1980.

[9] G. J. Foschini and J. Salz, "Digital communications over fading radio channels," Bell Syst. Tech. J., vol. 62, pp. 429-456, Feb. 1983.

[10] J. H. Winters, "Switched diversity with feedback for DPSK mobile radio systems," IEEE Trans. Veh. Technol., vol. VT-32, pp. 134-150, Feb. 1983

Jack H. Winters (S'77-M'81-SM'88-F'96) received the B.S.E.E. degree from the University of Cincinnati, Cincinnati, OH, in 1977 and the M.S. and the Ph.D. degrees in electrical engineering from The Ohio State University, Columbus, in 1978 and 1981, respectively.

He has been with AT\&T Bell Laboratories, now AT\&T Labs-Research, since 1981, where he is in the Wireless Systems Research Department. He has studied signal processing techniques for increasing the capacity and reducing signal distortion in fiber optic, mobile radio, and indoor radio systems, and is currently studying adaptive arrays and equalization for indoor and mobile radio.

Dr. Winters is a member of Sigma Xi.

Jack Salz (S'59-M'89) received the B.S.E.E. degree in 1955, the M.S.E. degree in 1956, and the Ph.D. degree in 1961, all in electrical engineering, from the University of Florida, Gainesville, FL.

He joined AT\&T Bell Laboratories in 1956, where he first worked on the electronic switching system. From 1968 to 1981, he supervised a group engaged in theoretical work in data communications. During the academic year 1967-1968, he was on leave from AT\&T Bell Laboratories as a Professor of electrical engineering at the University of Florida. In the spring of 1981, he was a Visiting Lecturer at Stanford University, Stanford, CA. In the spring of 1983, he was a Mackay Lecturer at the University of California, Berkeley. In 1988, he held the Shirly and Burt Harris Chair in Electrical Engineering at Technion-Israel Institute of Technology, Haifa, Israel. In 1992, he became an AT\&T Bell Laboratories Fellow. He retired from AT\&T in 1995 and is currently splitting his time between Lucent-Bell Labs, Bellcore, the University of California at Berkeley, and the Technion. 